\title{
Front-end Monitoring of Multiple Loads in Wireless Power Transfer
}

\section{Systems Without Wireless Communication Systems}

\author{
Jian Yin, Deyan Lin, Member, IEEE, Chi Kwan Lee, Member, IEEE, \\ Thomas Parisini, Fellow, IEEE and S.Y.(Ron) Hui, Fellow, IEEE
}

\begin{abstract}
This paper describes a method for monitoring multiple loads from the front-end of a wireless power transfer system without using any wireless communication systems. A mathematical approach based on scanning the frequency around the resonant frequency has been developed for deriving the load conditions. The proposal requires only information of the input voltage and current only, therefore eliminating the requirements of using wireless communication systems for feedback control. The proposal has been practically confirmed in hardware prototype with acceptable results.
\end{abstract}

Index Terms-Load monitoring, wireless power transfer, tesla's resonators.

\section{INTRODUCTION}

$\mathrm{N}$ icola Tesla investigated the fundamental principles of wireless power transfer based on the magnetic resonance and near-field coupling a century ago [1]. With the availability of power electronics as an enabling technology, wireless power transfer has re-emerged as a hot research topic in recent years, covering biomedical implant devices [2]-[10], inductive power transfer [11]-[13] and wireless charging techniques [14]-[19]. Due to the wireless nature of the system, wireless communication system has been proposed to feed back the load measurements for closed-loop control purpose [20]. However, it is preferable to remove this wireless communication link between the transmitter coil and the receiver coil in order to reduce system cost and complexity. A number of research efforts have been attempted to implement load detection methods on the transmitter side in a two-coil wireless power transfer (WPT) system with a single load [21]-[26]. Recently, reference [27] shows that it is possible to estimate the load variation based on the measurement of input voltage and current in a single load system. In some applications, there is a need for powering multiple loads simultaneously from the

Manuscript received January 19, 2015; revised March 11, 2015; accepted April 14, 2015. This work was supported by the Hong Kong Research Grant Council under GRF Project HKU 712913E.

J. Yin, D. Lin, and C. K. Lee are with the Department of Electrical and Electronic Engineering, The University of Hong Kong, Hong Kong (e-mail: jyin@eee.hku.hk; deyanlin@eee.hku.hk; cklee@eee.hku.hk).

T. Parisini is with the Department of Electrical and Electronic Engineering, Imperial College London, London SW7 2AZ, U.K. He is also with the Department of Engineering and Architecture, University of Trieste, Trieste, 34127, Italy (e-mail: t.parisini@gmail.com).

S. Y. R. Hui is with the Department of Electrical and Electronic Engineering, The University of Hong Kong, Hong Kong. He is also with the Department of Electrical and Electronic Engineering, Imperial College London, London SW7 2AZ, U.K. (e-mail: ronhui@eee.hku.hk). power source. Therefore WPT systems consisting of multiple receiver coils have been investigated in recent years [28]-[33].

Two camps of wireless power, namely the Wireless Power Consortium (WPC) and the Alliance for Wireless Power (A4WP) have been targeting wireless charging of multiple devices and wireless charging with spatial freedom. For the WPC, one of the major milestones for Version 1.2 of the Qi standard is "multi-device V.1.2 receivers charging on a V.1.2 transmitter with one inverter, delivering lowest cost designs" [34]. For the Rezence standard promoted by the A4WP, multi-device charging is highlighted as a technical specification [35]. In fact, wireless charging pads for multiple devices have been launched commercially. An example of a wireless charging pad capable of charging up to 5 devices simultaneously is shown in Fig.1.

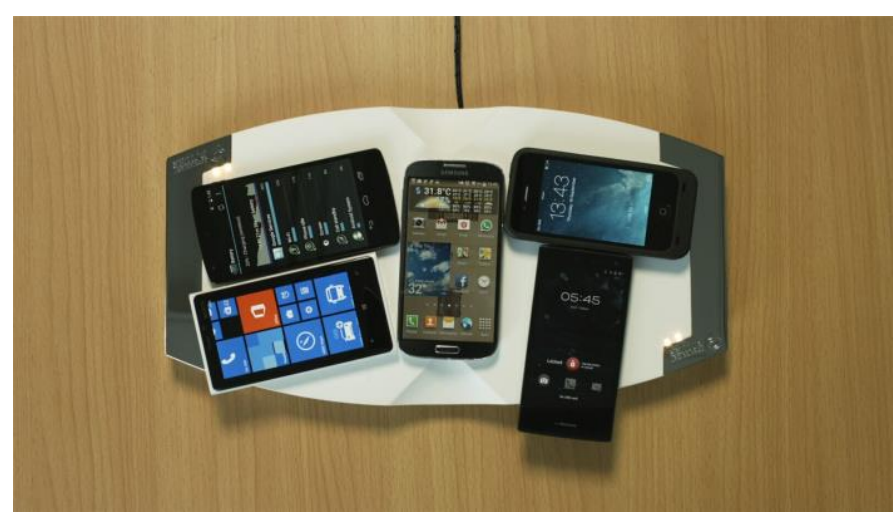

Fig. 1 An example of wireless charging pad for multiple devices (courtesy of ConvenientPower Ltd.).

In this project, a novel computational load estimation method is reported and demonstrated in a WPT system with two loads. The proposed method can manage nonlinear and time-varying input information in order to determine the multiple-load conditions without using any direct output feedback from the loads. This work is an extended version of [36], and it includes new implementation details and practical measurements under static and dynamic load conditions. This method is simple and time-efficient especially for large systems with several magnetic resonators and longer transmission distance. This computational approach is explained and practically verified with measurements obtained from a 4-coil WPT system under both static and dynamic load conditions. The proposal can greatly reduce the system cost and complexity by eliminating the requirements of any wireless communication systems that are often used in WPT systems for output feedback purposes [20],[37]. 


\section{MATHEMATICAL MODEL}

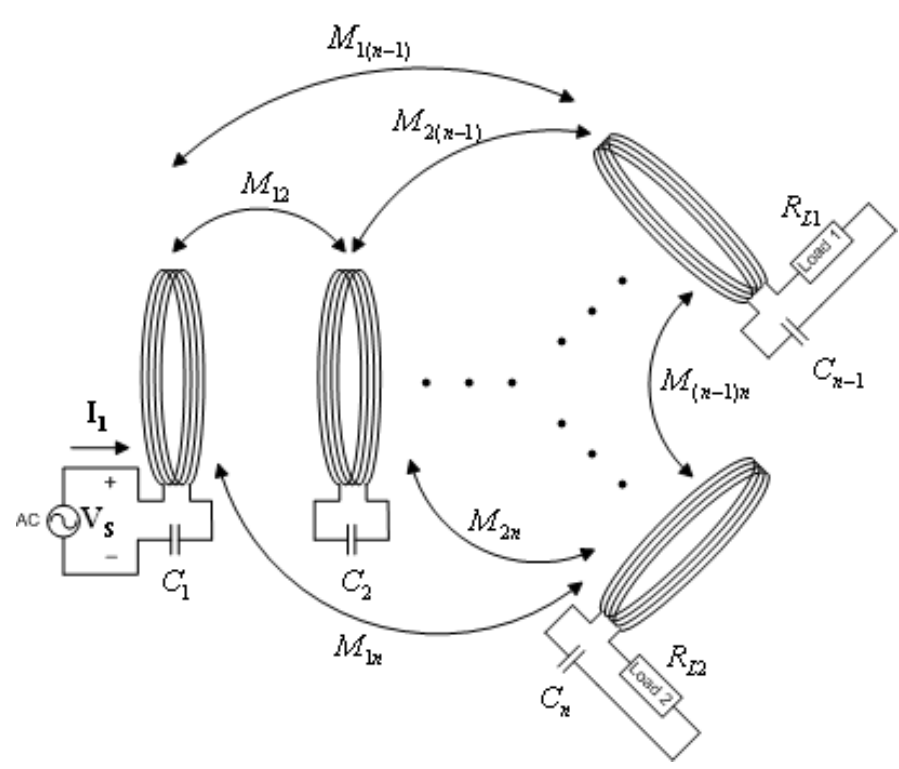

Fig. 2 An n-coil wireless power transfer system with two loads.

Fig. 2 illustrates a general WPT system consisting of $n$ coils, where an AC sinusoidal voltage source with a root-mean-square amplitude $\mathbf{V}_{\mathbf{s}}$ and an angular frequency $\omega$ is applied to the transmitter coil-1 on the input side. Two load resistors $R_{L 1}$ and $R_{L 2}$ are connected respectively to coil- $(n-1)$ and coil- $n$ on the output sides. In principle, the two loads can be connected to any pair of coils in the system except the transmitter coil-1. In this case, the coil-1 is the transmitter coil, the coil- $(n-1)$ and coil- $n$ are the receiver coils. The other coils with indices from 2 to $n-2$ are the relay coils. A capacitor is connected in series with each coil to form a resonator so as to enhance the power transfer capability. The resonant frequency of each coil resonator should match the input frequency of the power source. Since every coil resonator forms a closed circuit loop itself, by applying the Kirchhoff's Voltage Law to the $n$ coils the system matrix equation can be expressed as (1).

$$
\left[\begin{array}{c}
\mathbf{V}_{\mathbf{s}} \\
0 \\
\vdots \\
0 \\
0
\end{array}\right]=\left[\begin{array}{ccccc}
Z_{1} & j \omega M_{12} & \cdots & j \omega M_{1(n-1)} & j \omega M_{1 n} \\
j \omega M_{12} & Z_{2} & \cdots & j \omega M_{2(n-1)} & j \omega M_{2 n} \\
\vdots & \vdots & \ddots & \vdots & \vdots \\
j \omega M_{1(n-1)} & j \omega M_{2(n-1)} & \cdots & Z_{n-1}+R_{L 1} & j \omega M_{(n-1) n} \\
j \omega M_{1 n} & j \omega M_{2 n} & \cdots & j \omega M_{(n-1) n} & Z_{n}+R_{L 2}
\end{array}\right]
$$

$$
\times\left[\begin{array}{c}
\mathbf{I}_{1} \\
\mathbf{I}_{2} \\
\vdots \\
\mathbf{I}_{\mathrm{n}-1} \\
\mathbf{I}_{\mathbf{n}}
\end{array}\right]
$$

where $Z_{i}=R_{i}+j\left(\omega L_{i}-\frac{1}{\omega C_{i}}\right)$ is the total impedance, $R_{i}$ is the equivalent resistance, $L_{i}$ is the self-inductance, and $C_{i}$ is the capacitance of the coil- $i ; \mathbf{I}_{\mathbf{i}}$ is the current phasor flowing in the coil- $i ; M_{i j}$ is the mutual inductance between the coil- $i$ and coil $-j\left(M_{i j}=M_{j i}\right), \quad 1 \leq i \leq n, 1 \leq j \leq n$.

Some assumptions are made in the following analysis:

- All the parameters of the coils can be measured or calculated. Since the positions of coils are fixed, the mutual inductance of every pair of coils is also constant and can be measured accurately or calculated mathematically. (Note: this assumption can be justified as a patent-pending technique [38] has been developed for real-time identification of system parameters including the mutual inductance terms in the system matrix of (1).)

- The input voltage phasor $\mathbf{V}_{\mathbf{S}}$ and current phasor $\mathbf{I}_{\mathbf{1}}$ in the coil- 1 can be measured in real time.

\section{COMPUTATIONAL APPROACH FOR DOUBLE LOAD APPLICATION}

\section{A. Steps to Re-arrange the System Equations}

Now (1) can be re-arranged in the following steps:

$\left\{\begin{array}{c}\mathbf{V}_{\mathbf{S}}=Z_{1} \mathbf{I}_{1}+j \omega M_{12} \mathbf{I}_{2}+\cdots+j \omega M_{1 n} \mathbf{I}_{\mathbf{n}} \\ 0=j \omega M_{12} \mathbf{I}_{1}+Z_{2} \mathbf{I}_{2}+\cdots+j \omega M_{2 n} \mathbf{I}_{\mathbf{n}} \\ \vdots \\ 0=j \omega M_{1(n-1)} \mathbf{I}_{1}+j \omega M_{2(n-1)} \mathbf{I}_{2}+\cdots+j \omega M_{(n-1) n} \mathbf{I}_{\mathbf{n}}+R_{L 1} \mathbf{I}_{\mathbf{n}-\mathbf{1}} \\ 0=j \omega M_{1 n} \mathbf{I}_{1}+j \omega M_{2 n} \mathbf{I}_{2}+\cdots+Z_{n} \mathbf{I}_{\mathbf{n}}+R_{L 2} \mathbf{I}_{\mathbf{n}}\end{array}\right.$

The unknowns in above equations are $\mathbf{I}_{2}, \mathbf{I}_{3}, \cdots, \mathbf{I}_{\mathbf{n}}, R_{L 1}$ and $R_{L 2}$. By transferring all the known terms (which are the terms with $\mathbf{I}_{1}$ ) from the right hand side of (2) to the left hand side and leaving only unknown terms on the right hand side, (2) can be re-written as (3).

$$
\left\{\begin{array}{c}
\mathbf{V}_{\mathbf{S}}-Z_{1} \mathbf{I}_{1}=j \omega M_{12} \mathbf{I}_{2}+\cdots+j \omega M_{1(n-1)} \mathbf{I}_{\mathbf{n}-\mathbf{1}}+j \omega M_{1 n} \mathbf{I}_{\mathbf{n}} \\
-j \omega M_{12} \mathbf{I}_{1}=Z_{2} \mathbf{I}_{2}+\cdots+j \omega M_{2(n-1)} \mathbf{I}_{\mathbf{n}-\mathbf{1}}+j \omega M_{2 n} \mathbf{I}_{\mathbf{n}} \\
\vdots \\
-j \omega M_{1(n-1)} \mathbf{I}_{1}=j \omega M_{2(n-1)} \mathbf{I}_{2}+\cdots+j \omega M_{(n-1) n} \mathbf{I}_{\mathbf{n}}+Z_{L 1} \mathbf{I}_{\mathbf{n}-\mathbf{1}} \\
-j \omega M_{1 n} \mathbf{I}_{1}=j \omega M_{2 n} \mathbf{I}_{2}+\cdots+Z_{n} \mathbf{I}_{\mathbf{n}}+Z_{L 2} \mathbf{I}_{\mathbf{n}}
\end{array}\right.
$$

Equation (3) is nonlinear because it consists of the products of two unknowns $R_{L 1} \mathbf{I}_{\mathbf{n}-1}$ and $R_{L 2} \mathbf{I}_{\mathbf{n}}$. If one regard the terms $R_{L 1} \mathbf{I}_{\mathbf{n}-1}$ and $R_{L 2} \mathbf{I}_{\mathbf{n}}$ as two new unknowns, the original $n+1$ unknowns (i.e. $\mathbf{I}_{2}, \mathbf{I}_{3}, \cdots, \mathbf{I}_{\mathbf{n}}, R_{L 1}$ and $R_{L 2}$ ) will become $\mathbf{I}_{2}, \mathbf{I}_{3}, \cdots, \mathbf{I}_{\mathbf{n}}, \quad R_{L 1} \mathbf{I}_{\mathbf{n}-1}$ and $R_{L 2} \mathbf{I}_{\mathbf{n}}$. Then (3) can be transformed into a linear form as shown in (4). However the solutions to (4) are not unique, because there are $n$ equations and $n+1$ unknowns. By putting the term $R_{L 1} \mathbf{I}_{\mathbf{n}-1} \quad$ on the left hand side of the equation in the form of (5), then the unknowns of (5) can be placed on the left hand side of (6). 


$$
\left[\begin{array}{c}
\mathbf{V}_{\mathbf{S}}-Z_{1} \mathbf{I}_{1} \\
-j \omega M_{12} \mathbf{I}_{1} \\
\vdots \\
-j \omega M_{1(n-1)} \mathbf{I}_{1} \\
-j \omega M_{1 n} \mathbf{I}_{1}
\end{array}\right]=
$$$$
\left[\begin{array}{cccccc}
j \omega M_{12} & j \omega M_{13} & \cdots & j \omega M_{1 n} & 0 & 0 \\
Z_{2} & j \omega M_{23} & \cdots & j \omega M_{2 n} & 0 & 0 \\
\vdots & \vdots & \ddots & \vdots & \vdots & \vdots \\
j \omega M_{2(n-1)} & j \omega M_{3(n-1)} & \cdots & j \omega M_{(n-1) n} & 1 & 0 \\
j \omega M_{2 n} & j \omega M_{3 n} & \cdots & Z_{n} & 0 & 1
\end{array}\right] \times\left[\begin{array}{c}
\mathbf{I}_{2} \\
\vdots \\
\mathbf{I}_{\mathbf{n}} \\
R_{L 1} \mathbf{I}_{\mathbf{n}-1} \\
R_{L 2} \mathbf{I}_{\mathbf{n}}
\end{array}\right]
$$

$$
\left[\begin{array}{c}
\mathbf{V}_{\mathbf{s}}-Z_{1} \mathbf{I}_{1} \\
-j \omega M_{12} \mathbf{I}_{\mathbf{1}} \\
\vdots \\
-j \omega M_{1(n-1)} \mathbf{I}_{\mathbf{1}}-R_{L 1} \mathbf{I}_{\mathbf{n}-1} \\
-j \omega M_{1 n} \mathbf{I}_{1}
\end{array}\right]=
$$$$
\left[\begin{array}{ccccc}
j \omega M_{12} & j \omega M_{13} & \cdots & j \omega M_{1 n} & 0 \\
Z_{2} & j \omega M_{23} & \cdots & j \omega M_{2 n} & 0 \\
\vdots & \vdots & \ddots & \vdots & \vdots \\
j \omega M_{2(n-1)} & j \omega M_{3(n-1)} & \cdots & j \omega M_{(n-1) n} & 0 \\
j \omega M_{2 n} & j \omega M_{3 n} & \cdots & Z_{n} & 1
\end{array}\right] \times\left[\begin{array}{c}
\mathbf{I}_{2} \\
\mathbf{I}_{\mathbf{3}} \\
\vdots \\
\mathbf{I}_{\mathbf{n}} \\
R_{L 2} \mathbf{I}_{\mathbf{n}}
\end{array}\right]
$$

$$
\left[\begin{array}{c}
\mathbf{I}_{2} \\
\mathbf{I}_{3} \\
\vdots \\
\mathbf{I}_{\mathbf{n}} \\
R_{L 2} \mathbf{I}_{\mathbf{n}}
\end{array}\right]=\mathbf{P} \times\left[\begin{array}{c}
V_{S}-Z_{1} \mathbf{I}_{1} \\
-j \omega M_{12} \mathbf{I}_{1} \\
\vdots \\
-j \omega M_{1(n-1)} \mathbf{I}_{1}-R_{L 1} \mathbf{I}_{\mathbf{n}-1} \\
-j \omega M_{1 n} \mathbf{I}_{1}
\end{array}\right]
$$

where $\mathbf{P}$ is the inverse matrix of the coefficient matrix in (5) and

$\mathbf{P}=\left[\begin{array}{ccccc}j \omega M_{12} & j \omega M_{13} & \cdots & j \omega M_{1 n} & 0 \\ Z_{2} & j \omega M_{23} & \cdots & j \omega M_{2 n} & 0 \\ \vdots & \vdots & \ddots & \vdots & \vdots \\ j \omega M_{2(n-1)} & j \omega M_{3(n-1)} & \cdots & j \omega M_{(n-1) n} & 0 \\ j \omega M_{2 n} & j \omega M_{3 n} & \cdots & Z_{n} & 1\end{array}\right]^{-1}$

Note that the last 3 equations of the matrix equation (6) are:

$$
\begin{aligned}
& \mathbf{I}_{\mathbf{n}-\mathbf{1}}=a+b R_{L 1} \mathbf{I}_{\mathbf{n}-\mathbf{1}} \\
& \mathbf{I}_{\mathbf{n}}=c+d R_{L 1} \mathbf{I}_{\mathbf{n}-1} \\
& R_{L 2} \mathbf{I}_{\mathbf{n}}=e+f R_{L 1} \mathbf{I}_{\mathbf{n}-\mathbf{1}}
\end{aligned}
$$

where

$$
\begin{aligned}
& \left\{\begin{array}{l}
a=\mathbf{P}_{\mathbf{n}-2} \mathbf{V} \\
b=-p_{(n-2)(n-1)} \\
c=\mathbf{P}_{\mathbf{n}-\mathbf{1}} \mathbf{V} \\
d=-p_{(n-1)(n-1)} \\
e=\mathbf{P}_{\mathbf{n}} \mathbf{V} \\
f=-p_{n(n-1)}
\end{array}\right. \\
& \mathbf{V}=\left[\begin{array}{c}
\mathbf{V}_{\mathbf{S}}-Z_{1} \mathbf{I}_{\mathbf{1}} \\
-j \omega M_{12} \mathbf{I}_{\mathbf{1}} \\
\vdots \\
-j \omega M_{1(n-1)} \mathbf{I}_{\mathbf{1}} \\
-j \omega M_{1 n} \mathbf{I}_{\mathbf{1}}
\end{array}\right]
\end{aligned}
$$

$\mathbf{P}_{\mathbf{i}}$ is the $i$-th row vector in matrix $\mathbf{P}$, and $p_{i j}$ is the element on the $i$-th row and $j$-th column in the $\mathbf{P}$ matrix. From (8),

$$
\mathbf{I}_{\mathbf{n}-1}=\frac{a}{1-b R_{L 1}}
$$

Substituting (13) into (9),

$$
\mathbf{I}_{\mathbf{n}}=c+\frac{a d R_{L 1}}{1-b R_{L 1}}
$$

Substituting (13) and (14) into (10), the $R_{L 1}-R_{L 2}$ relationship is:

$$
A R_{L 1} R_{L 2}+B R_{L 1}+C R_{L 2}+D=0
$$

where,

$$
\left\{\begin{array}{l}
A=a d-b c=\alpha_{A}+j \beta_{A} \\
B=b e-a f=\alpha_{B}+j \beta_{B} \\
C=c=\alpha_{C}+j \beta_{C} \\
D=-e=\alpha_{D}+j \beta_{D}
\end{array}\right.
$$

Here the four coefficients $A, B, C, D$ are complex numbers whereas the two unknowns $R_{L 1}$ and $R_{L 2}$ are real. By comparing the coefficients of the real and the imaginary parts in (15), it can be shown that:

$$
\begin{aligned}
& \alpha_{A} R_{L 1} R_{L 2}+\alpha_{B} R_{L 1}+\alpha_{C} R_{L 2}=-\alpha_{D} \\
& \beta_{A} R_{L 1} R_{L 2}+\beta_{B} R_{L 1}+\beta_{C} R_{L 2}=-\beta_{D}
\end{aligned}
$$

With some mathematical manipulations of (17) and (18) to eliminate the nonlinear term $R_{L 1} R_{L 2}$,

$$
\left(\alpha_{B} \beta_{A}-\alpha_{A} \beta_{B}\right) R_{L 1}+\left(\alpha_{C} \beta_{A}-\alpha_{A} \beta_{C}\right) R_{L 2}=\alpha_{A} \beta_{D}-\alpha_{D} \beta_{A}
$$

In (19), there are two independent unknowns $R_{L 1}$ and $R_{L 2}$ with real coefficients related to the measurements of $\mathbf{V}_{\mathbf{S}}$ and $\mathbf{I}_{\mathbf{1}}$ for one specific frequency. If the frequency is changed to another value, a new set of coefficients will be obtained. Theoretically, only two sets of $\mathbf{V}_{\mathbf{S}}$ and $\mathbf{I}_{\mathbf{1}}$ measurements obtained at two different frequencies are sufficient in order to 
calculate the two load resistance values, provided that there is no parameter tolerance and measurement errors. In practice, one cannot avoid measurement noise and error. It will be shown that data sets obtained from more than two different frequencies are needed in order to reduce the estimation errors.

\section{B. A Typical Case: Three-Coil System}

The proposed method can be illustrated with a three-coil WPT system where the coil-1 is the transmitter. The two loads $R_{L 1}$ and $R_{L 2}$ are connected to coil-2 and coil-3 respectively. For such a system, the inverse matrix $\mathbf{P}$ in the form of (7) can be expressed as (20)

$$
\begin{aligned}
& \mathbf{P}= \\
& {\left[\begin{array}{ccc}
\frac{M_{23}}{M_{12}\left(j \omega M_{23}-\frac{M_{13} Z_{2}}{M_{12}}\right.} & -\frac{\frac{M_{13}}{M_{12}}}{j \omega M_{23}-\frac{M_{13} Z_{2}}{M_{12}}} & 0 \\
-\frac{Z_{2}}{j \omega M_{12}\left(j \omega M_{23}-\frac{M_{13} Z_{2}}{M_{12}}\right)} & \frac{1}{j \omega M_{23}-\frac{M_{13} Z_{2}}{M_{12}}} & 0 \\
\frac{Z_{2} Z_{3}-\left(j \omega M_{23}\right)^{2}}{j \omega M_{12}\left(j \omega M_{23}-\frac{M_{13} Z_{2}}{M_{12}}\right)} & -\frac{j \omega M_{13} M_{23}}{Z_{12}} & 1 \\
j \omega M_{23}-\frac{M_{13} Z_{2}}{M_{12}} &
\end{array}\right]}
\end{aligned}
$$

The results of (20) are essential to the proposed calculation procedure. For example, the intermediate coefficients $a, b, c, d$, $e, f$ in (11) and $A, B, C, D$ in (16) are obtained with the help of (20). Finally the two load resistances in (19) can be found.

\section{Least Square Approximation}

In order to overcome the problems arising from the measurement errors and parameter tolerance, it is necessary to use more than two sets of measured input voltage and current data obtained at different frequencies. Equation (19) can be described as a straight line in an x-y coordinate plane. Imagine a general multiple-coil WPT system with two loads (say both $10 \mathrm{Ohms}$ ). If it is under the ideal condition that the system parameters are and the input measurements of $V_{\mathbf{S}}$ and $\mathbf{I}_{1}$ are accurate, all the $R_{L 1}-R_{L 2}$ lines described by (19) for different input frequencies should intersect at one point as shown in Fig. 3. Such single intersection point should provide the unique solutions for (19). In practice, there are tolerance in systems parameters and noise in measurements. In this case the $R_{L 1}-R_{L 2}$ lines described by (19) for different input frequencies will have multiple intersection points as shown in Fig. 4. So one cannot rely on the use of data sets obtained from only two frequencies to find the correct solution. In this study, the least square method is used to obtain the solution with improved accuracy.

The least square theorem [39] can be described as follows: let $\mathbf{A}$ be a $m \times n$ matrix, the least square solution to the system $\mathbf{A} \hat{\mathbf{x}}=\mathbf{b}$ can be expressed as

$$
\hat{\mathbf{x}}=\left(\mathbf{A}^{\mathrm{T}} \mathbf{A}\right)^{-1} \mathbf{A}^{\mathrm{T}} \mathbf{b}
$$

where $\hat{\mathbf{x}}$ is the column matrix having $n$ elements, and $\mathbf{b}$ is the column matrix consisting of $m$ elements. With respect to (19) in this study,

$\mathbf{A}=\left[\begin{array}{cc}\alpha_{B 1} \beta_{A 1}-\alpha_{A 1} \beta_{B 1} & \alpha_{C 1} \beta_{A 1}-\alpha_{A 1} \beta_{C 1} \\ \alpha_{B 2} \beta_{A 2}-\alpha_{A 2} \beta_{B 2} & \alpha_{C 2} \beta_{A 2}-\alpha_{A 2} \beta_{C 2} \\ \vdots & \vdots \\ \alpha_{B m} \beta_{A m}-\alpha_{A m} \beta_{B m} & \alpha_{C m} \beta_{A m}-\alpha_{A m} \beta_{C m}\end{array}\right]$

$\hat{\mathbf{x}}=\left[\begin{array}{l}R_{L 1} \\ R_{L 2}\end{array}\right]$

$\mathbf{b}=\left[\begin{array}{c}\alpha_{A 1} \beta_{D 1}-\alpha_{D 1} \beta_{A 1} \\ \alpha_{A 2} \beta_{D 2}-\alpha_{D 2} \beta_{A 2} \\ \vdots \\ \alpha_{A m} \beta_{D m}-\alpha_{D m} \beta_{A m}\end{array}\right]$

The number of equations used is $m$. The geometric meaning of the least square operation here is to find an optimum equivalent point among all the intersection points of the $m$ straight lines. Such point should have the nearest distance to all of the $m$ straight lines.

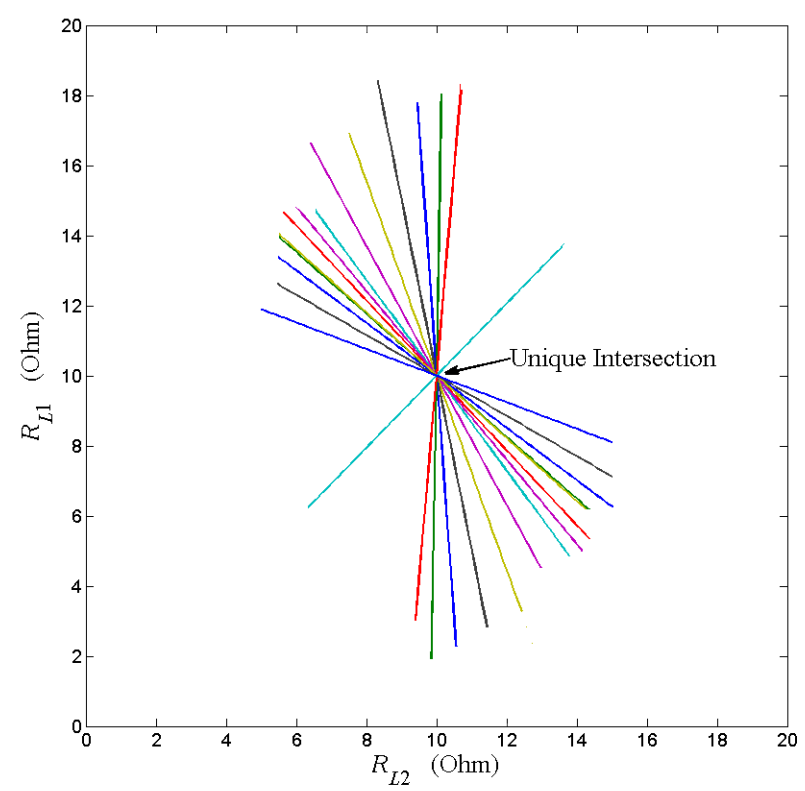

Fig. 3 Unique intersection under ideal condition. 


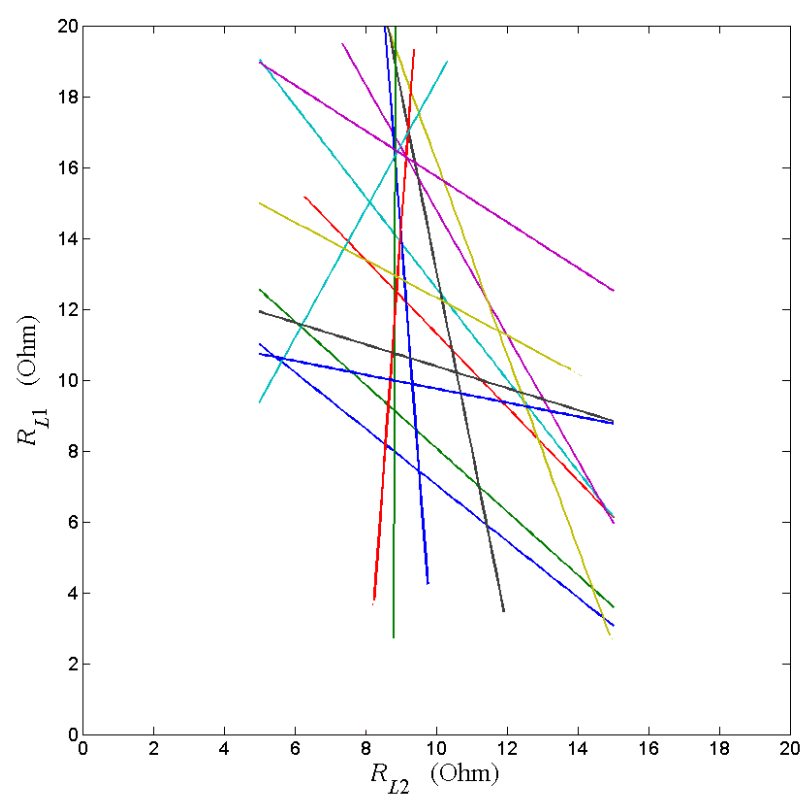

Fig. 4 Multiple intersections under practical conditions.

\section{An Exceptional Case}

Theoretically, it seems that this method cannot work under the conditions of a symmetric geometrical structure and identical load circuit parameters. Take a three-coil system depicted in Fig. 5 as an example. The coil-1 is the transmitter coil. If two identical receiver coil resonators (i.e. coil-2 and coil-3) are located symmetrically with respect to the coil-1, then $M_{12}=M_{13}, R_{2}=R_{3}, L_{2}=L_{3}$ and $C_{2}=C_{3}$. If the loads $R_{L 1}$ and $R_{L 2}$ are connected to coil-2 and coil-3 respectively, a pair of input voltage and current measurements can be obtained in the coil-1. If the two loads are interchanged, i.e. connecting $R_{L 1}$ to the coil-3 and $R_{L 2}$ to the coil-2, there will not be any change in the input voltage and current. Thus under such situation, although a unique pair of load solutions for $R_{L 1}$ and $R_{L 2}$ can still be obtained, one cannot tell which value is for $R_{L 1}$ and which for $R_{L 2}$. Therefore in practical applications, the symmetric structure such as the one in Fig. 5 should be avoided in the system design stage.

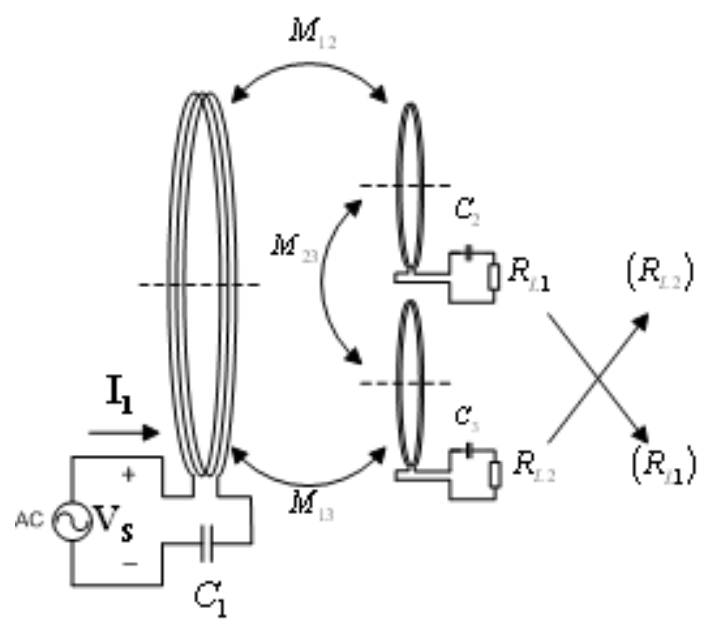

Fig. 5 A symmetric 3-coil wireless power transfer system with two loads.

\section{EXPERIMENTAL VERIFICATION}

In order to verify the proposed computational method for identifying the load resistance values, a 4-coil wireless power domino-resonator system is adopted in the experimental study. Each coil has the same parameters $\left(R_{1 \sim 4}=0.6788 \Omega, L_{1} \sim\right.$ ${ }_{4}=82.03 \mu \mathrm{H}$ ). The coil diameter is $30 \mathrm{~cm}$, and the number of turns is 11 . The details of the system parameters are provided in Tables I and II. The system setup is illustrated in Fig. 6. The four coils are aligned along one direction with even distance of about $10 \mathrm{~cm}$. The first coil is the transmitter, the coil-3 is connected to one load $R_{L 1}$, and the coil-4 is connected to the other load $R_{L 2}$. The input voltage is supplied by the function generator Tektronix AFG 3102. The input voltage and current are captured by the oscilloscope Agilent OSO-X 3034A. The instantaneous measured data are transferred to the computer which calculates the load resistance values.

Table I Mutual-inductance of each pair of coils of the 4-coil domino-resonator system.

\begin{tabular}{|c|c|c|c|c|c|c|}
\hline & $\boldsymbol{M}_{\mathbf{1 2}}$ & $\boldsymbol{M}_{\mathbf{1 3}}$ & $\boldsymbol{M}_{\mathbf{1 4}}$ & $\boldsymbol{M}_{\mathbf{2 3}}$ & $\boldsymbol{M}_{\mathbf{2 4}}$ & $\boldsymbol{M}_{\mathbf{3 4}}$ \\
\hline $\begin{array}{c}\text { Inductance } \\
(\boldsymbol{\mu H})\end{array}$ & 15.45 & 5.863 & 2.645 & 15.43 & 5.769 & 15.13 \\
\hline
\end{tabular}

Table II Capacitance Value of each series capacitor of the 4-coil domino-resonator system.

\begin{tabular}{|c|c|c|c|c|}
\hline & $\boldsymbol{C}_{\mathbf{1}}$ & $\boldsymbol{C}_{\mathbf{2}}$ & $\boldsymbol{C}_{\mathbf{3}}$ & $\boldsymbol{C}_{\mathbf{4}}$ \\
\hline $\begin{array}{c}\text { Capacitance } \\
(\mathrm{nF})\end{array}$ & 1.029 & 1.024 & 1.013 & 1.046 \\
\hline
\end{tabular}




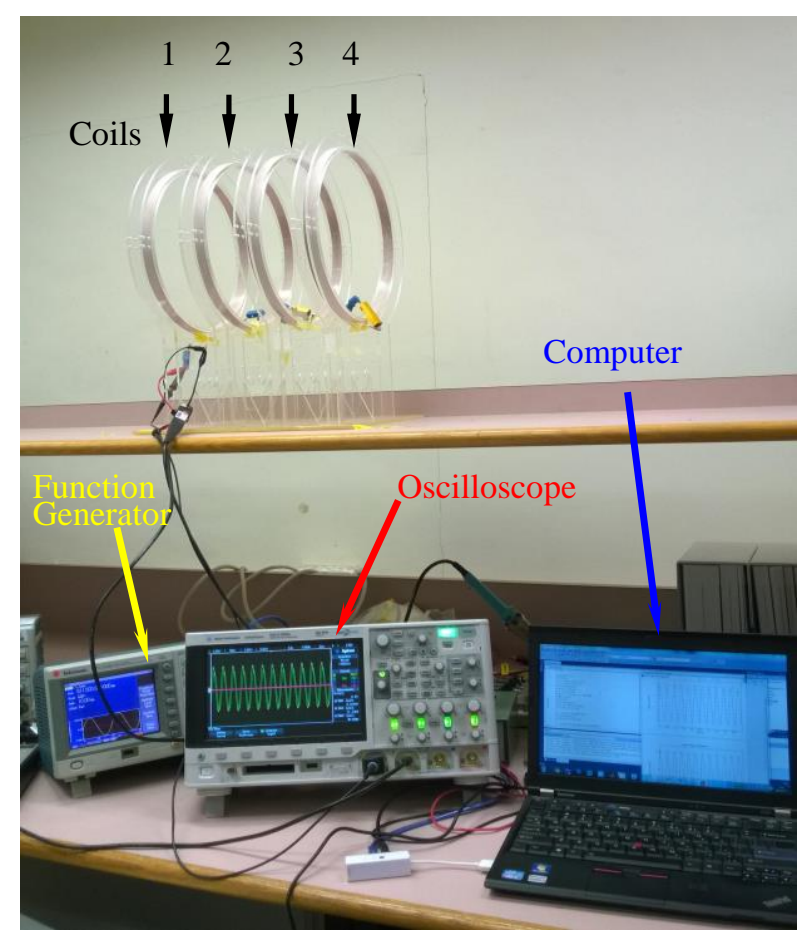

Fig. 6 Experiment setup.

\section{A. Constant Load Detection}

Tests have been conducted by connecting two resistors $R_{L 1}$ and $R_{L 2}$ to the coil-3 and coil-4 respectively. By sweeping the input frequency from $470 \mathrm{kHz}$ to $630 \mathrm{kHz}$ to estimate the resistor values using the least square method, Fig. 7 illustrates the calculated load values based on the data sets obtained from 2 to 40 frequency scans for three different load resistance combinations (i.e. (i) $R_{L 1}=0 \mathrm{Ohms}, R_{L 2}=10 \mathrm{Ohms}$; (ii) $R_{L 1}=$ $10 \mathrm{Ohms}, R_{L 2}=10 \mathrm{Ohms}$; (iii) $R_{L 1}=50 \mathrm{Ohms}, R_{L 2}=20 \mathrm{Ohms}$ ). When the information from only two frequencies is applied to find the load solutions, the results are far from satisfactory. This observation highlights the effects of the parameter tolerance (error) and measurement noise. The least square method enables the solutions to converge to the reference values when more data sets obtained from the frequency scans are used. The number of frequency scans for accurate estimations depends on the practical system configuration. In this study, reasonably accurate values of the load resistance can be obtained with estimated values calculated from 20 different frequencies.

The time for calculating the load values can be estimated. In general, five cycles of measurements are used for each frequency scan. For the frequency range from $470 \mathrm{kHz}$ to 630 $\mathrm{kHz}$, the time for 5 cycles of an average frequency of $500 \mathrm{kHz}$ is only $40 \mu \mathrm{s}$. So it takes typically less than $50 \mu$ s to obtain the required data sets for load estimation. To solve the equations of this method, typical computational time by microprocessors is less than a few milliseconds (depending on the type of processor). For the load monitoring of multiple loads under static charging, the load conditions do not change quickly. If the load monitoring is carried out every 3 seconds, for example, the time of a few milliseconds required for checking the load conditions is negligible.

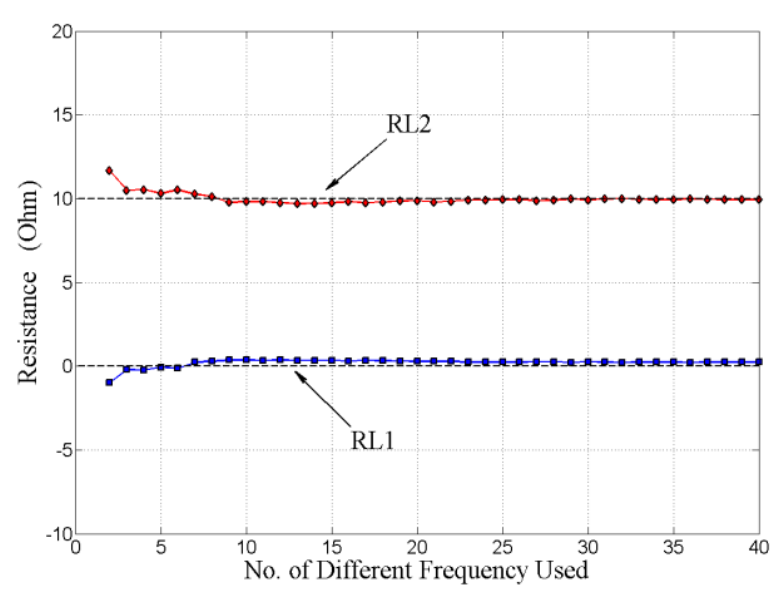

(a) RL1=0, RL2=10 Ohms.

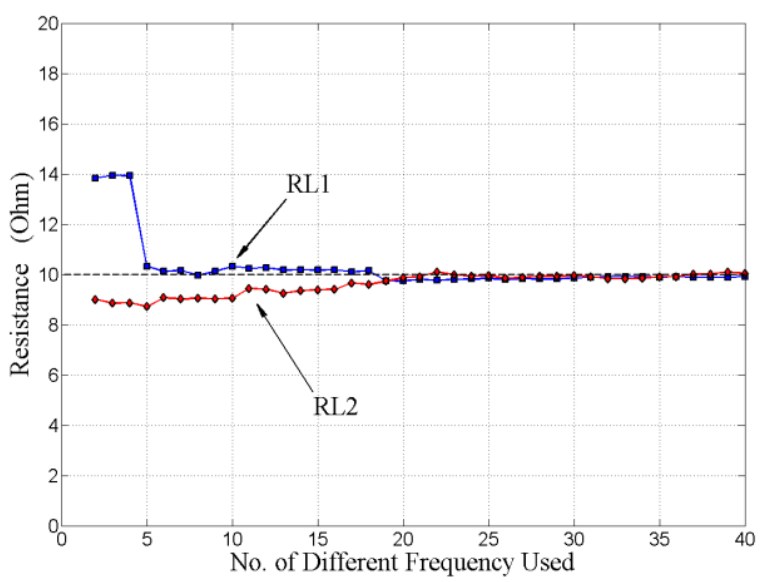

(b) RL1=RL2=10 Ohms.

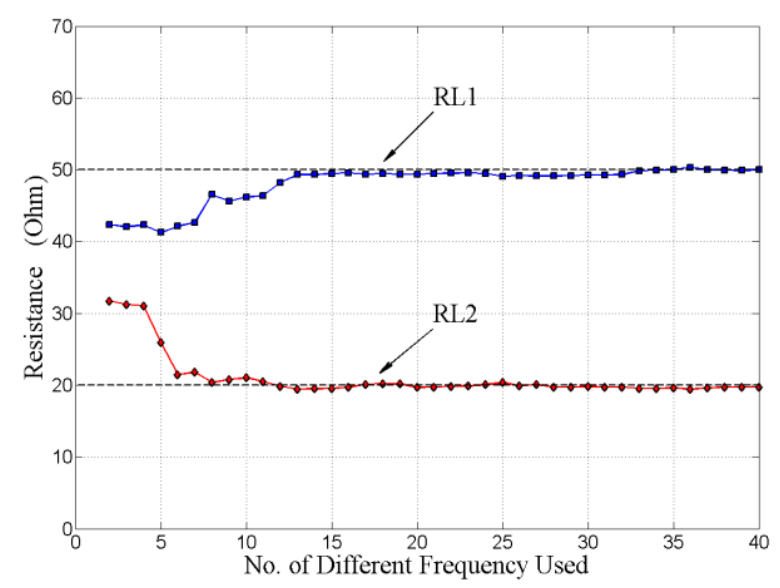

(c) RL1=50, RL2=20 Ohms.

Fig. 7 Estimated load resistances using more than two frequencies. 


\section{B. Variable Load Detection}

Another set of tests have been conducted by using two variable resistors connected to the coil-3 and coil-4. For each frequency scan, the measured waveforms of input voltage and current are transmitted to the computer so that the amplitudes and phase difference can be calculated. Then the $R_{L 1}-R_{L 2}$ equation (19) can be solved with data obtained from different frequencies. When the threshold number of the frequency scans is reached, the solutions should converge to the reference values.

Fig. 8 shows the estimated load results when one of the two resistive loads is kept constant and the other is varied in steps with time. The dotted curves represent the reference values, and the solid curves represent the estimated resistance values. Here the threshold number of adopted frequencies is 20. It can be observed that there is a transient time period when the estimated load value is changing from one to another level. The less data sets are used, the shorter the transient time period becomes. However if the number of data sets is not sufficient, the calculated resistor values will be far from the reference values.

Fig. 9 depicts the new case where both of the load resistors are changing and the estimated load resistances are calculated with 30 frequency scans. As more equations are involved, the transient time in Fig. 9 is longer than that in Fig. 8. However the load curves in Fig. 9 are smoother than those in Fig. 8 because more data are used now. In the middle of the test in Fig. $9, R_{L 1}$ is deliberately reduced to zero (i.e. to simulate a short circuit fault at the terminals of the coil-3). The estimated results of $R_{L I}$ in Fig.9 indicate that the proposed approach can detect such conditions in a WPT system.

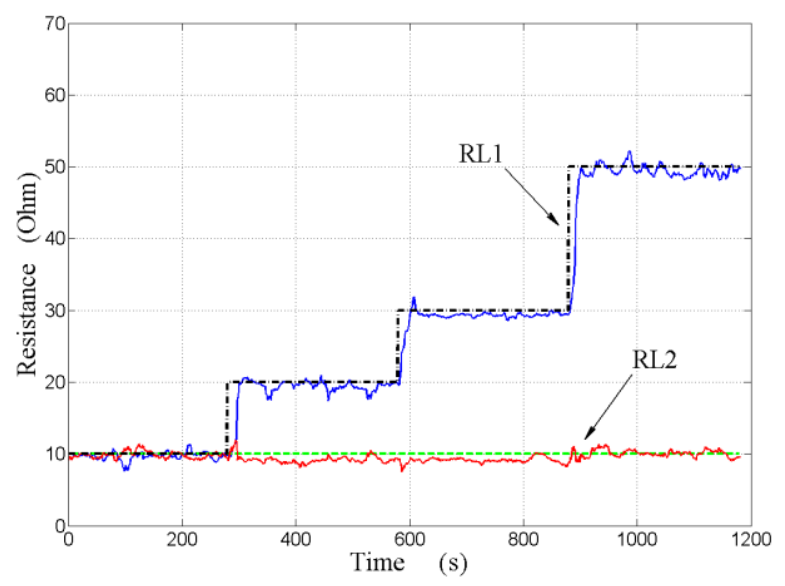

Fig. 8 Calculated resistances with 20 equations when load is changing.

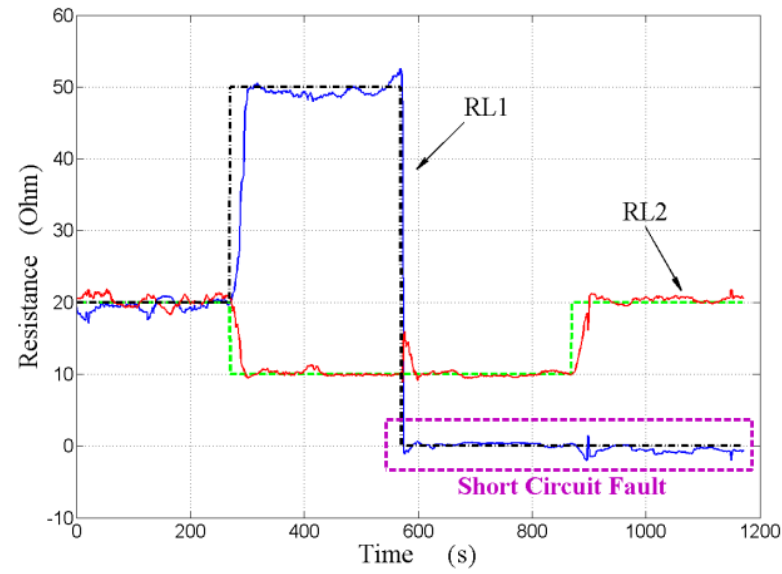

Fig. 9 Calculated resistances with 30 equations when load is changing.

\section{CONCLUSION}

In this paper, a new method is presented for monitoring two loads in a wireless power transfer system, using only the input voltage and input current measurements. The method overcomes the errors introduced by the parameter tolerance and measurement noise by solving the proposed equation set with a number of frequency scans and using the least square approximation method to obtain the estimated load values with reasonably good accuracy. This approach has the advantage of eliminating the need for a wireless communication system on the receiver side of the system, therefore reducing the cost and circuit complexity of the receiver circuits in wireless power systems. The proposed method has been practically demonstrated in a 4-coil wireless power transfer system with two loads of various combinations of resistance values. For the experimental setup with four coils and two loads, it is found that about 20 sets of data (obtained at 20 different frequencies around the resonant frequency of the resonators) are sufficient to obtain results with good accuracy. Of course, the speed of the implementation depends on the speed of the processor. In this study, the input voltage and current waveforms are sampled by a digital oscilloscope and the sampled data are exported to an external computer for monitoring the load resistance values. If faster response is required for other purpose such as real-time control, the input voltage and current can be captured directly by a fast processor. Since the time required for the frequency scans and calculating the load values is typically less than a few milliseconds, the proposed method will not affect the time for wireless power transfer significantly even if the load monitoring is applied regularly.

\section{REFERENCES}

[1] N. Tesla, "Apparatus for transmitting electrical energy," U.S. Patent 1119 732, Dec. 1, 1914.

[2] G. B. Joung and B. H. Cho, "An energy transmission system for an artificial heart using leakage inductance compensation of transcutaneous transformer," IEEE Trans. Power Electron., vol. 
13, no. 6 , pp. $1013-1022$, Nov. $1998 .$.

[3] G. Wang, W. Liu, M. Sivaprakasam, and G. A. Kendir, "Design and analysis of an adaptive transcutaneous power telemetry for biomedical implants," IEEE Trans. Circuits Syst. I, Reg. Papers, vol. 52, no. 10, pp. 2109-2117, Oct. 2005.

[4] P. Si, A. P. Hu, J. W. Hsu, M. Chiang, Y. Wang, S. Malpas, and D. Budgett, "Wireless power supply for implantable biomedical device based on primary input voltage regulation," in Proc. 2nd IEEE Conf. Ind. Electron. Appl. (ICIEA), May 2007, pp. 235-239.

[5] P. Si, A. P. Hu, S. Malpas, and D. Budgett, "A frequency control method for regulating wireless power to implantable devices," IEEE Trans. Biomed. Circuits Syst., vol. 2, no. 1, pp. 22-29, Mar. 2008.

[6] K. RamRakhyani, S. Mirabbasi, and M. Chiao, "Design and optimization of resonance-based efficient wireless power delivery systems for biomedical implants," IEEE Trans. Biomed. Circuits Syst., vol. 5, no. 1, pp. 48-63, Feb. 2011.

[7] X. Luo, S. Nui, S. L. Ho, and W. N. Fu, "A design method of magnetically resonanting wireless power delivery systems for bio-implantable devices," IEEE Trans. Magn., vol. 47, no. 10, pp. 3833-3836, Oct. 2011

[8] Y. Y. Ko, S. L. Ho, W. N. Fu, and X. Zhang, "A novel hybrid resonator for wireless power delivery in bio-implantable devices," IEEE Trans. Magn., vol. 48, no. 11, pp. 4518-4521, Nov. 2012.

[9] H. Jiang, J. Zhang, D. Lan, K. K. Chao, S. Liou, H. Shahnasser, R. Fechter, S. Hirose, M. Harrison, and S. Roy, "A low-frequency versatile wireless powertransfer technology for biomedical implants," IEEE Trans. Biomed. Circuits Syst., vol. 7, no. 4, pp. 526-535, Aug. 2013.

[10] D. Ahn, and S. Hong, "Wireless power transmission with self-regulated output voltage for biomedical implant," IEEE Trans. Ind. Electron., vol. 61, no. 5, pp. 2225-2235, May 2014.

[11] A. W. Green, and J. T. Boys, " $10 \mathrm{kHz}$ inductively coupled power transfer-concept and control," in Proc.5th Int. Conf. Power Electron. Variable Speed-Drives, Oct. 1994, pp. 694-699.

[12] J. T. Boys, G. A. Covic, and A. W. Green, "Stability and control of inductively coupled power transfer systems," IEE Proc.-Electr. Power Appl., vol. 147, no. 1, pp. 37-43, Jan. 2000.

[13] G. A. J. Elliott, G. A. Covic, D. Kacprzak, and J. T. Boys, “A New Concept: Asymmetrical Pick-Ups for Inductively Coupled Power Transfer Monorail Systems," IEEE Trans. Magn., vol. 42, no. 10, pp. 3389-3391, Oct. 2006.

[14] Y. Jang, and M. M. Jovanovic, "A contactless electrical energy transmission system for portable-telephone battery chargers," IEEE Trans. Ind. Electron., vol. 50, no. 3, pp. 520-527, Jun. 2003.

[15] B. Choi, J. Nho, H. Cha, T. Ahn, and S. Choi, "Design and implementation of low-profile contactless battery charger using planar printed circuit board windings as energy transfer device," IEEE Trans. Ind. Electron., vol. 51, no. 1, pp. 140-147, Feb. 2004.

[16] S. Y. R. Hui, and W. C. Ho, "A new generation of universal contactless battery charging platform for portable consumer electronic equipment," IEEE Trans. Power Electron., vol. 20, no. 3, pp. 620-627, May 2005.

[17] X. Liu, and S. Y. R. Hui, "Simulation study and experimental verification of a universal contactless battery charging platform with localized charging features," IEEE Trans. Power Electron., vol. 22, no. 6, pp. 2202-2210, Nov. 2007.

[18] X. Liu, and S. Y. R. Hui, "Optimal design of a hybrid winding structure for planar contactless battery charging platform," IEEE Trans. Power Electron., vol. 23, no. 1, pp. 455-463, Jan. 2008.

[19] S. Y. Hui, "Planar wireless charging technology for portable electronic products and qi," Proc. IEEE, vol. 101, no. 6, pp. 1290-1301, Jun. 2013.

[20] N. Y. Kim, K. Y. Kim, J. Choi, and C. W. Kim, "Adaptive frequency with power-level tracking system for efficient magentic resonance wireless power transfer," Electron. Lett., vol. 48, no. 8, pp. 452-454, Apr. 2012.

[21] U. K. Madawala, and D. J. Thrimawithana, "A single controller for inductive power transfer systems," in Proc. IEEE IECON, Nov. 2009, pp. 109-113.

[22] D. J. Thrimawithana, and U. K. Madawala, "A primary side controller for inductive power transfer systems," in Proc. IEEE Int. Conf. Ind. Technol. (ICIT), Mar. 2010, pp. 661-666.

[23] U. K. Madawala, and D. J. Thrimawithana, "New technique for inductive power transfer using a single controller," IET Power Electron., vol. 5, no. 2, pp. 248-256, Feb. 2012.

[24] X. Dai, Y. Sun, C. Tang, Z. Wang, Y. Su, and Y. Li, "Dynamic parameters identification method for inductively coupled power transfer system," in Proc. IEEE Int. Conf. Sustainable Energy Technol. (ICSET), Dec. 2010, pp. 1-5.

[25] Z. Wang, X. Lv, Y. Sun, X. Dai, and Y. Li, “A simple approach for load identification in current-fed inductive power transfer system," in Proc. IEEE Int. Conf. Power Syst. Technol. (POWERCON), Oct./Nov. 2012, pp. 1-5.

[26] Z. Wang, Y. Li, Y. Sun, C. Tang, and X. Lv, "Load detection model of voltage-fed inductive power transfer system," IEEE Trans. Power Electron., vol. 28, no. 11, pp. 5233-5243, Nov. 2013.

[27] J. Yin, D. Lin, C. K. Lee, and S. Y. R. Hui, "A systematic approach for load monitoring and power control in wireless power transfer systems without any direct output measurement," IEEE Trans. Power Electron., vol. 30, no. 3, pp. 1657-1667, Mar. 2015.

[28] B. L. Cannon, J. F. Hoburg, D. D. Stancil, and S. C. Goldstein, "Magnetic resonant coupling as a potential means for wireless power transfer to multiple small receivers," IEEE Trans. Power Electron., vol. 24, no. 7, pp. 1819-1825, Jul. 2009.

[29] J. J. Casanova, Z. N. Low, and J. Lin, "A loosely coupled planar wireless power system for multiple receivers," IEEE Trans. Ind. Electron., vol. 56, no. 8, pp. 3060-3068, Aug. 2009.

[30] I. Awai, K. Yamaguchi, T. Ishida, and T. Ishizaki, "Design of a resonator-coupled wpt system with multiple loads based on measurement with a vna instead of an oscilloscope," in Proc. Asia-Pacific Microwave Conf.(APMC), Dec. 2012, pp. 824-826.

[31] F. F. A. van der Pijl, M. Castilla, and P. Bauer, "Adaptive sliding-mode control for a multiple-user inductive power transfer system without need for communication," IEEE Trans. Ind. Electron., vol. 60 , no. 1, pp. 271-279, Jan. 2013.

[32] D. Ahn, and S. Hong, "Effect of coupling between multiple transmitters or multiple receivers on wireless power transfer," IEEE Trans. Ind. Electron., vol. 60 , no. 7, pp. 2602-2613, Jul. 2013.

[33] T. Zhang, M. Fu, C. Ma, and X. Zhu, "Optimal load analysis for a two-receiver wireless power transfer system," in Proc. IEEE Wireless Power Transfer Conf. (WPTC), May 2014, pp. 84-87.

[34] T. Francesca, "Major milestones for V1.2 resonant specification", Wireless Power Consortium, [online] http://www.wirelesspowerconsortium.com/blog/86/major-milest ones-for-v12-resonant-specification, March 2015

[35] [online] http://www.rezence.com/technology/meet-rezence, March 2015

[36] J. Yin, D. Lin, C. K. Lee, and S. Y. R. Hui, "Monitoring of multiple loads in wireless power transfer systems without direct output feedback," in Proc. IEEE Appl. Power Electron. Conf. Expo. (APEC), Mar. 2014, pp. 1165-1170.

[37] O. C. Onar, J. M. Miller, S. L. Campbell, C. Coomer, C. P. White, and L. E. Seiber, "Oak ridge national laboratory wireless power transfer development for sustainable campus initiative," in Proc. IEEE Transportation Electrification Conf. Expo. (ITEC), Jun. 2013, pp. 1-8.

[38] S. Y. R. Hui, D. Lin, J. Yin, and C. K. Lee, "Method for parameter identification, load monitoring and output power control of wireless power transfer systems," US patent application, US 61/862,627, 6 August, 2013.

[39] G. Strang, Introduction to Linear Algebra. Wellesley, MA: 
Wellesley Cambridge Press, 1998.

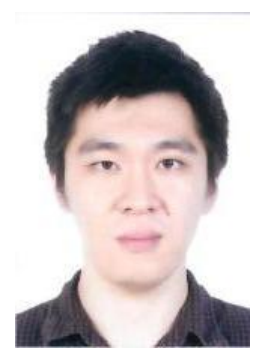

Jian Yin was born in Jinan, China, in 1984. He received the B.E. degree from Shandong University, China, in 2007. During 2007 to 2009 he worked as an Electrical Engineer in SDEPCI, China, to design power substations. Currently he is working toward the Ph.D. degree with the Department of Electrical and Electronic Engineering, The University of Hong Kong, Hong Kong. His research interests include sensorless motor drives and wireless power

transfer technologies.

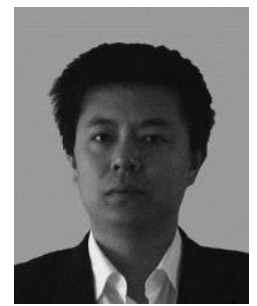

Deyan Lin (M'09) was born in China, in 1972. $\mathrm{He}$ received the B.Sc. and M.A.Sc. degrees from Huazhong University of Science and Technology, Wuhan, China, in 1995 and 2004, respectively, and the Ph.D. degree from the City University of Hong Kong, Kowloon, in 2012. He is currently a Research Associate with the Department of Electrical and Electronic Engineering, The University of Hong Kong, Pokfulam. From 1995 to 1999, he was a Teaching Assistant in the Electrical Engineering Department at Jianghan University, Wuhan, where he became a Lecturer later. From 2008 to 2009, he was a Senior Research Assistant with the City University of Hong Kong. His current research interests include memristors, and modeling, control, simulation of gas-discharge lamps, and wireless power transfer.

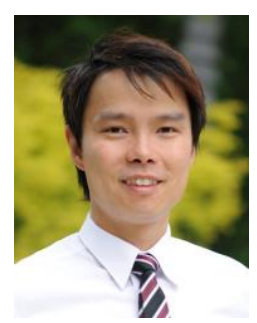

Chi Kwan Lee (M'08-SM'14) received the B.Eng. and Ph.D. degrees in electronic engineering from the City University of Hong Kong, Kowloon, Hong Kong, in 1999 and 2004, respectively. He was a Postdoctoral Research Fellow in the Power and Energy Research Centre at the National University of Ireland, Galway, from 2004 to 2005. In 2006, he joined the Centre of Power Electronics in City University of Hong Kong as a Research Fellow. From 2008-2011 he was a Lecturer of Electrical Engineering at the Hong Kong Polytechnic University. He was a Visiting Academic at Imperial College London from 2010-2013. Since January 2012, he has been an Assistant Professor at the Department of Electrical \& Electronic Engineering, The University of Hong Kong. His current research interests include applications of power electronics to power systems, advanced inverters for renewable energy and smart grid applications, reactive power control for load management in renewable energy systems, wireless power transfer, energy harvesting, and planar electromagnetics for high frequency power converters.

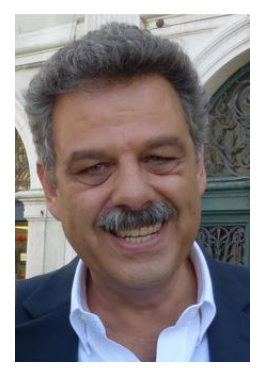

Thomas Parisini (S'87-M'88-SM'99-F'11) received the Ph.D. degree in Electronic Engineering and Computer Science in 1993 from the University of Genoa. He was with Politecnico di Milano and since 2010 he holds the Chair of Industrial Control at Imperial College London. Since 2001 he is also Danieli Endowed Chair of Automation Engineering with University of Trieste. In 2009-2012 he was Deputy Rector of University of Trieste. He authored or co-authored more than 250 research papers in archival journals, book chapters, and international conference proceedings. His research interests include neural-network approximations for optimal control problems, fault diagnosis for nonlinear and distributed systems, nonlinear model predictive control systems and nonlinear estimation. He is a co-recipient of the IFAC Best Application Paper Prize of the Journal of Process Control, Elsevier, for the three-year period 2011-2013 and of the 2004 Outstanding Paper Award of the IEEE Trans. on Neural Networks. He is also a recipient of the 2007 IEEE Distinguished Member Award. He is involved as Project Leader in several projects funded by the European Union, by the Italian Ministry for Research, and he is currently leading consultancy projects with some major process control companies (Danieli, Duferco, Electrolux, among others). In 2012 he was awarded a prestigious ABB Research Grant dealing with energy-autonomous sensor networks for self-monitoring industrial environments. Thomas Parisini is the Editor-in-Chief of the IEEE Trans. on Control Systems Technology. He is also the Chair of the IFAC Technical Committee on Fault Detection, Supervision \& Safety of Technical Processes SAFEPROCESS. He was a Distinguished Lecturer and an elected member of the Board of Governors of the IEEE Control Systems Society. Among other activities, he was the Program Chair of the 2008 IEEE Conference on Decision and Control and the General Co-Chair of the 2013 IEEE Conference on Decision and Control. Prof. Parisini is a Fellow of the IEEE.

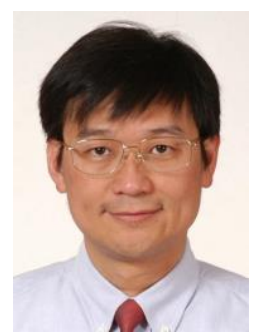

S. Y. (Ron) Hui (M'87-SM'94-F'03) received his B.Sc. (Eng,) (Hons.) degree at the University of Birmingham in 1984 and a D.I.C. and Ph.D. degree at Imperial College London in 1987. Presently, he holds the Philip Wong Wilson Wong Chair Professorship at the University of Hong Kong and a Chair Professorship at Imperial College London. He has published over 300 technical papers, including more than 180 refereed journal publications. Over 55 of his patents have been adopted by industry. He is an Associate Editor of the IEEE Transactions on Power Electronics and IEEE Transactions on Industrial Electronics, and an Editor of the IEEE Journal of Emerginig and Selected Topics in Power Electronics. He was appointed twice as an IEEE Distinguished Lecturer by the IEEE Power Electronics Society in 2004 and 2006, he won an IEEE Best Paper Award from the IEEE IAS in 2002, and two IEEE Power Electronics Transactions Prize Paper Awards in 2009 and 2010. His inventions on wireless charging platform technology underpin key dimensions of Qi, the world's first wireless power standard, with freedom of positioning and localized charging features for wireless charging of consumer electronics. In November 2010, he received the IEEE Rudolf Chope R\&D Award from the IEEE Industrial Electronics Society and the IET Achievement Medal (The Crompton Medal). He is a Fellow of the Australian Academy of Technological Sciences \& Engineering and is the recipient of the 2015 IEEE William E. Newell Power Electronics Award. 